\title{
Herschel-SPIRE spectroscopy of the DR21 molecular cloud core
}

\author{
G. J. White ${ }^{1,2}$, A. Abergel ${ }^{4}$, L. Spencer ${ }^{3}$, N. Schneider ${ }^{5}$, D. A. Naylor ${ }^{3}$, L.D. Anderson ${ }^{8}$, C. Joblin ${ }^{17,18}$, P. Ade ${ }^{7}$,
} P. André ${ }^{5}$, H. Arab ${ }^{4}$, J.-P. Baluteau ${ }^{8}$, J.-P. Bernard ${ }^{17,18}$, K. Blagrave ${ }^{9}$, S. Bontemps ${ }^{5,6}$, F. Boulanger ${ }^{4}$, M. Cohen ${ }^{10}$, M. Compiegne ${ }^{9}$, P. Cox ${ }^{11}$, E. Dartois ${ }^{4}$, G. Davis ${ }^{12}$, R. Emery ${ }^{2}$, T. Fulton ${ }^{4}$, B. Gom ${ }^{4}$, M. Griffin ${ }^{7}$, C. Gry ${ }^{8}$, E. Habart ${ }^{4}$, M. Huang ${ }^{14}$, S. Jones ${ }^{3}$, J. M. Kirk ${ }^{7}$, G. Lagache ${ }^{4}$, S. Leeks ${ }^{2}$, T. Lim² ${ }^{2}$ S. Madden 5 , G. Makiwa ${ }^{3}$, P. Martin 9 , M.-A. Miville-Deschênes ${ }^{4}$, S. Molinari ${ }^{14}$, H. Moseley ${ }^{15}$, F. Motte ${ }^{5}$, K. Okumura ${ }^{5}$, D. Pinheiro Gonçalves ${ }^{9}$, E. Polehampton ${ }^{2,4}$, T. $\operatorname{Rodet}^{16}$, J. A. Rodón ${ }^{8}$, D. Russeil ${ }^{8}$, P. Saraceno ${ }^{14}$, S. Sidher ${ }^{2}$, B. M. Swinyard ${ }^{2}$, D. Ward-Thompson ${ }^{7}$, A. Zavagno ${ }^{8}$

(Affiliations are available in the online edition)

Received 31 March 2010 / Accepted 11 May 2010

\section{ABSTRACT}

We present far-infrared spectra and maps of the DR21 molecular cloud core between 196 and $671 \mu \mathrm{m}$, using the Herschel-SPIRE spectrometer. Nineteen molecular lines originating from $\mathrm{CO},{ }^{13} \mathrm{CO}, \mathrm{HCO}^{+}$and $\mathrm{H}_{2} \mathrm{O}$, plus lines of $[\mathrm{N} \mathrm{II}]$ and $[\mathrm{CI}]$ were recorded, including several transitions not previously detected. The CO lines are excited in warm gas with $T_{\text {kin }} \sim 125 \mathrm{~K}$ and $n_{\mathrm{H}_{2}} \sim 7 \times 10^{4} \mathrm{~cm}^{-3}$, CO column density $N(\mathrm{CO}) \sim 3.5 \times 10^{18} \mathrm{~cm}^{-2}$ and a filling factor of $\sim 12 \%$, and appear to trace gas associated with an outflow. The rotational temperature analysis incorporating observations from ground-based telescopes reveals an additional lower excitation $\mathrm{CO}$ compoment which has a temperature $\sim 78 \mathrm{~K}$ and $N(\mathrm{CO}) \sim 4.5 \times 10^{21} \mathrm{~cm}^{-2}$.

Key words. ISM: lines and bands - infrared: ISM - ISM: molecules

\section{Introduction}

We report observations of the far-IR spectrum of the DR21 molecular cloud core obtained with the Herschel satellite between 196 and $671 \mu \mathrm{m}$. The DR21 HII-region/molecular cloud is part of the Cygnus $\mathrm{X}$ complex of molecular clouds located at a distance of $1.7 \mathrm{kpc}$ (Schneider et al. 2006). This region has been subject to numerous studies at different wavelengths (Richardson et al. 1988; Wilson \& Mauersberger 1990; Liechti \& Walmsley 1997; Schneider et al. 2006, 2010; Jakob et al. 2007). The main DR21 cloud core has a mass of $\sim 20000 M_{\odot}$ (Richardson et al. 1989), and contains one of the most energetic star formation outflows detected, with an outflow mass of $\sim 3000 M_{\odot}$ (Garden et al. 1991; Cruz-González et al. 2010).

\section{SPIRE observations}

\subsection{Spectra}

We present science demonstration phase (SDP) observations obtained with ESA's Herschel Space Observatory (Pilbratt et al. 2010), using the Spectral and Photometric Imaging REceiver (SPIRE - Griffin et al. 2010). The calibration and characteristics of SPIRE have been described by Swinyard et al. (2010). SPIRE was operated as an imaging Fourier-transform spectrometer (FTS $)$ in the high resolution mode $\left(\lambda / \Delta \lambda=1000\left(=300 \mathrm{~km} \mathrm{~s}^{-1}\right.\right.$

* Herschel is an ESA space observatory with science instruments provided by European-led Principal Investigator consortia and with important participation from NASA. at $250 \mu \mathrm{m}$ ) sampling across an approximately circular field of view with an unvignetted diameter of $2.6^{\prime}$. This means that the line profiles are unresolved.

The sky footprint is formed by two detector arrays: the 19 pixel SLW array $(671-303 \mu \mathrm{m})$ and the 37 pixel SSW array $(313-194 \mu \mathrm{m})$, with beam widths varying from $17^{\prime \prime}$ at $194 \mu \mathrm{m}$ to $42^{\prime \prime}$ at $671 \mu \mathrm{m}$, with uncertainties of $\pm 7-10 \%$ (Griffin et al. 2010). The integration time was $1065 \mathrm{~s}$, summed from two seperate observations. The current best estimates of the absolute uncertainties for the FTS detectors are 10-20\% for the SSW detectors, and $\sim 30 \%$ for the SLW detectors (Swinyard et al. 2010).

The unapodised FTS spectra provide the highest spectral resolution, with a classical instrumental sinc function line shape. A spectral line fitting routine was developed for extracting line parameters (Jones et al. 2009). This fits a continuum (either a low order polynomial or a blackbody variant) using the LevenbergMarquardt least squares method. The fitting procedure weights the spectral intensity at a given frequency of an averaged spectrum by the statistical uncertainty at that frequency, returning line centers, intensities, line widths and their associated fit errors.

\subsection{Maps}

The SPIRE observations sparsely sample the field of view, although there are calibration uncertainties for the outer ring of detectors at the edges of both arrays that are not yet fully characterised. To provide a first look at the relative distributions in the various species, we have interpolated the fluxes of individual 

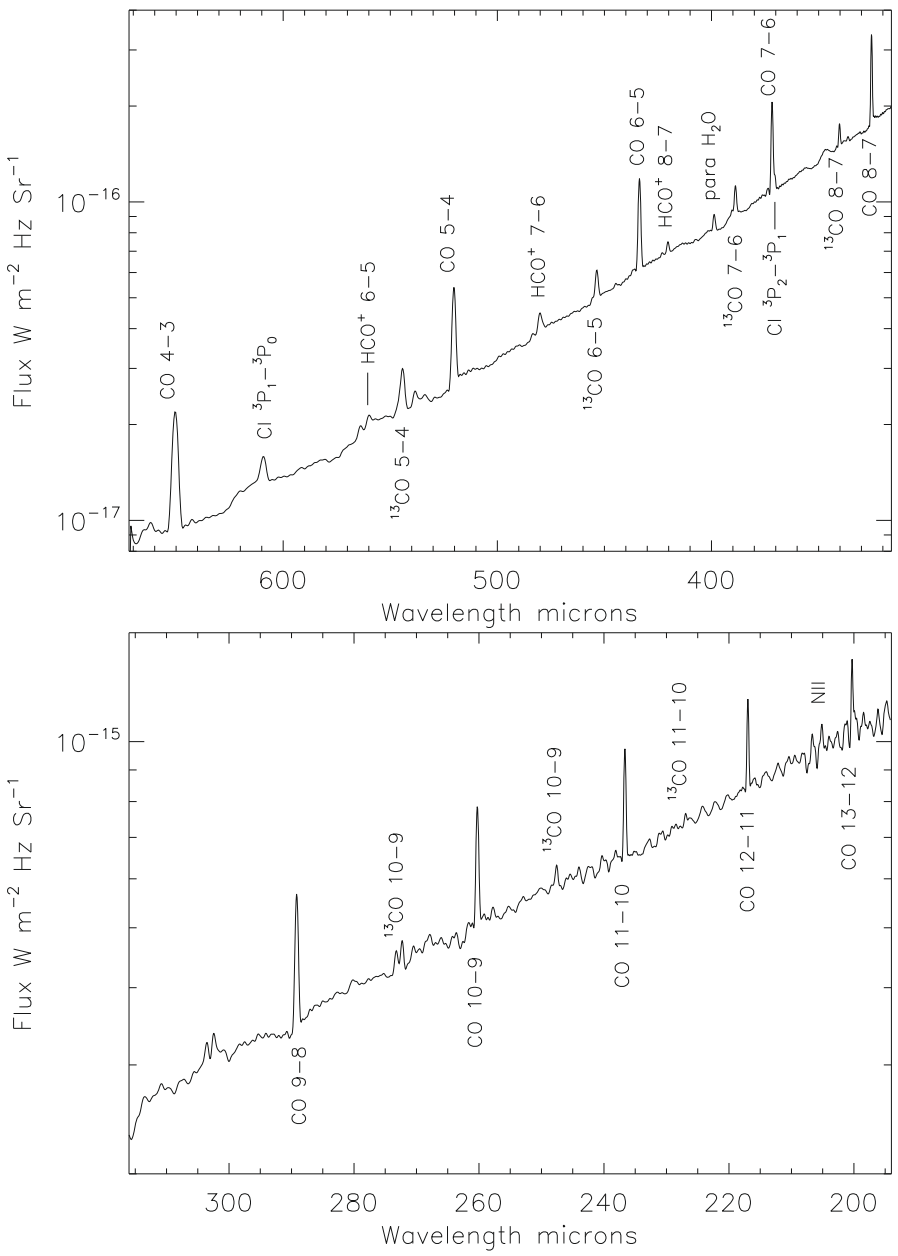

Fig. 1. (Upper picture) Spectrum of DR21 with the SLW detector array, with beam sizes varying from $42.0^{\prime \prime}$ at $671.1 \mu \mathrm{m}$ to $37.3^{\prime \prime}$ at $316.5 \mu \mathrm{m}$, and (lower picture) with the SSW detector array, with beam sizes varying from $18.7^{\prime \prime}$ at $281.7 \mu \mathrm{m}$ to $16.8^{\prime \prime}$ at $194.2 \mu \mathrm{m}$.

lines, although this spatial information is not used in subsequent line modeling.

The maps of selected species are shown in Fig. 2. The CO lines in both detector arrays show a prominent central peak, with extensions to the east and west along the well known outflow. This has been assumed to be associated with outflowing gas with $\left(T_{\mathrm{ex}} \sim 2000 \mathrm{~K}\right.$ and $H\left(\mathrm{H}_{2}\right) \sim 1 \times 10^{19} \mathrm{~cm}^{-2}$ from Garden et al. 1991). However, as will be seen in the high resolution JCMT observations (Fig. 3), the emission traced in the SPIRE maps is also clearly visible in the relatively low excitation $\mathrm{CO} J=3-2$ data, suggesting that there may be a mixture of low and high excitation gas present. This is confirmed in Fig. 3, where similar extensions of the ambient gas are present in the JCMT CO $J=3-2$ map, and that of Schneider et al. (2010). This is not unexpected, as this outflow appears to have a very large mass of several thousand $M_{\odot}$, and presumably the high velocity gas phase overlaps (or may co-exist with) ambient material. The SPIRE maps also show that the ${ }^{3} \mathrm{P}_{1}-{ }^{3} \mathrm{P}_{0}$ atomic carbon line has a similar spatial distribution to that of $\mathrm{CO}$. By contrast, the $\mathrm{H}_{2} \mathrm{O}$ and [N II] lines appear to be more compact and centred close to the DR21 cloud core, although the [N II] distribution is elongated to the east - observations with higher signal to noise and better sampling are needed for more detailed comparison.
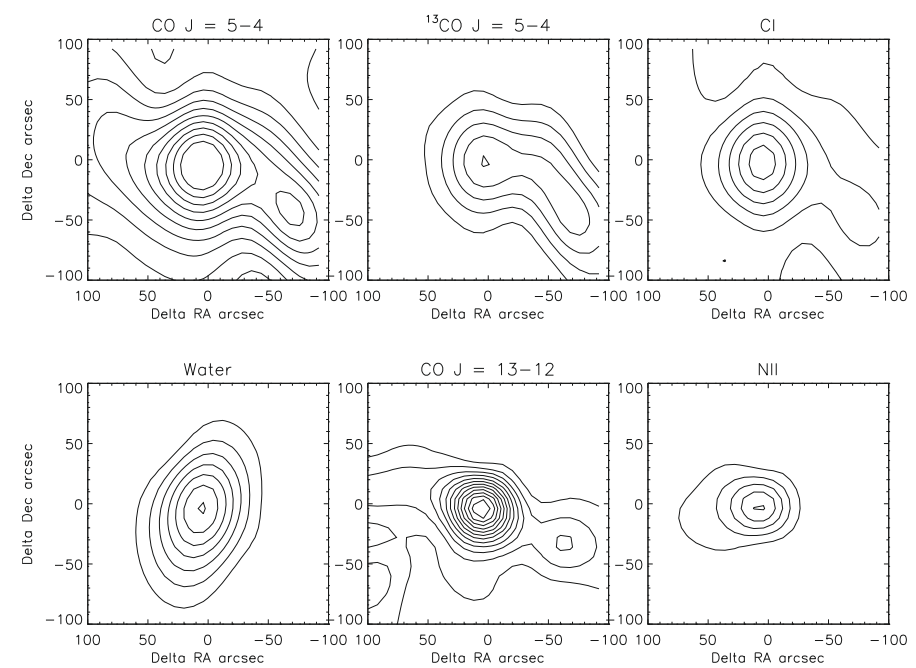

Fig. 2. The SPIRE maps of selected species toward DR21. Since the data points do not fully sample the mapped area, this map was constructed by interpolating between the data points. The $(0,0)$ position is located at RA $(2000)=20^{\mathrm{h}} 39^{\mathrm{m}} 01^{\mathrm{s}} .18$, Dec $(2000)=+42^{\circ} 19^{\prime} 43^{\prime \prime} .66$.

Table 1. Fluxes measured in the central pixel.

\begin{tabular}{lllll}
\hline \hline Species & Transition & $\begin{array}{c}\text { Wave } \\
\mu \mathrm{m}\end{array}$ & $\begin{array}{l}\text { Int intensity } \\
\mathrm{W} \mathrm{m}^{-2} \mathrm{sr}^{-1}\end{array}$ & $\begin{array}{l}\text { Intens error } \\
\mathrm{W} \mathrm{m}^{-2} \mathrm{sr}^{-1}\end{array}$ \\
\hline $\mathrm{CO}$ & $J=4-3$ & 650.1 & $2.85(-8)$ & $6.93(-10)$ \\
$\mathrm{CI}$ & ${ }^{3} \mathrm{P}_{1}-{ }^{3} \mathrm{P}_{0}$ & 609.0 & $4.86(-9)$ & $9.96(-10)$ \\
$\mathrm{HCO}^{+}$ & $J=6-5$ & 560.5 & $3.99(-9)$ & $4.29(-10)$ \\
${ }^{13} \mathrm{CO}$ & $J=5-4$ & 544.1 & $1.66(-8)$ & $5.04(-10)$ \\
$\mathrm{CO}$ & $J=5-4$ & 520.3 & $6.81(-8)$ & $3.39(-10)$ \\
$\mathrm{HCO}^{+}$ & $J=7-6$ & 480.3 & $1.02(-8)$ & $1.35(-9)$ \\
${ }^{13} \mathrm{CO}$ & $J=6-5$ & 453.5 & $2.44(-8)$ & $3.21(-9)$ \\
$\mathrm{CO}$ & $J=6-5$ & 433.5 & $1.15(-7)$ & $1.47(-8)$ \\
$\mathrm{HCO}$ & $J=8-7$ & 420.3 & $1.32(-8)$ & $2.10(-9)$ \\
$\mathrm{H}_{2} \mathrm{O}$ & $2{ }_{11}-202$ & 398.6 & $2.33(-8)$ & $3.03(-9)$ \\
${ }^{13} \mathrm{CO}$ & $J=7-6$ & 388.7 & $3.66(-8)$ & $5.88(-9)$ \\
$\mathrm{CO}$ & $J=7-6$ & 371.6 & $2.14(-7)$ & $1.29(-9)$ \\
$\mathrm{CI}$ & ${ }^{3} \mathrm{P}_{2}-{ }^{3} \mathrm{P}_{1}$ & 370.5 & $3.03(-8)$ & $1.26(-9)$ \\
${ }^{13} \mathrm{CO}$ & $J=8-7$ & 340.1 & $6.79(-8)$ & $1.80(-8)$ \\
$\mathrm{CO}$ & $J=8-7$ & 325.2 & $3.15(-7)$ & $4.56(-8)$ \\
$\mathrm{CO}$ & $J=9-8$ & 289.1 & $4.89(-7)$ & $4.23(-9)$ \\
$\mathrm{CO}$ & $J=10-9$ & 260.2 & $5.94(-7)$ & $1.01(-8)$ \\
$\mathrm{CO}$ & $J=11-10$ & 236.6 & $7.26(-7)$ & $5.46(-9)$ \\
$\mathrm{CO}$ & $J=12-11$ & 216.9 & $7.44(-7)$ & $6.72(-9)$ \\
$\mathrm{NII}$ & ${ }^{3} \mathrm{P}_{1}-{ }^{3} \mathrm{P}_{0}$ & 205.2 & $1.45(-7)$ & $4.71(-8)$ \\
$\mathrm{CO}$ & $J=13-12$ & 200.3 & $6.90(-7)$ & $3.96(-8)$ \\
\hline & & & &
\end{tabular}

\subsection{JCMT CO J = 3-2 observations}

CO $J=3-2$ JCMT archival data (programme M07AU01) with a $15^{\prime \prime}$ beam and spectral of $0.05 \mathrm{~km} \mathrm{~s}^{-1}$ are shown in Fig. 3, from a $4.5 \mathrm{~h}$ integration using the HARP array receiver. The area covered by the SPIRE footprint (Fig. 2) is shown as a white square. The JCMT observations clearly trace the outflow which runs from the NE-SW from DR21 from the centre of the white box. The JCMT map also reveals a prominent north-south ridge that includes $\mathrm{CO}$ peaks associated with the well-studied sources DR21 $(\mathrm{OH})$ and DR21-FIR1. Around the DR21 core, a bipolar structure close to the systemic velocity is coincident with distribution of high velocity gas and shocked $\mathrm{H}_{2}$ (Garden et al. 1991). 


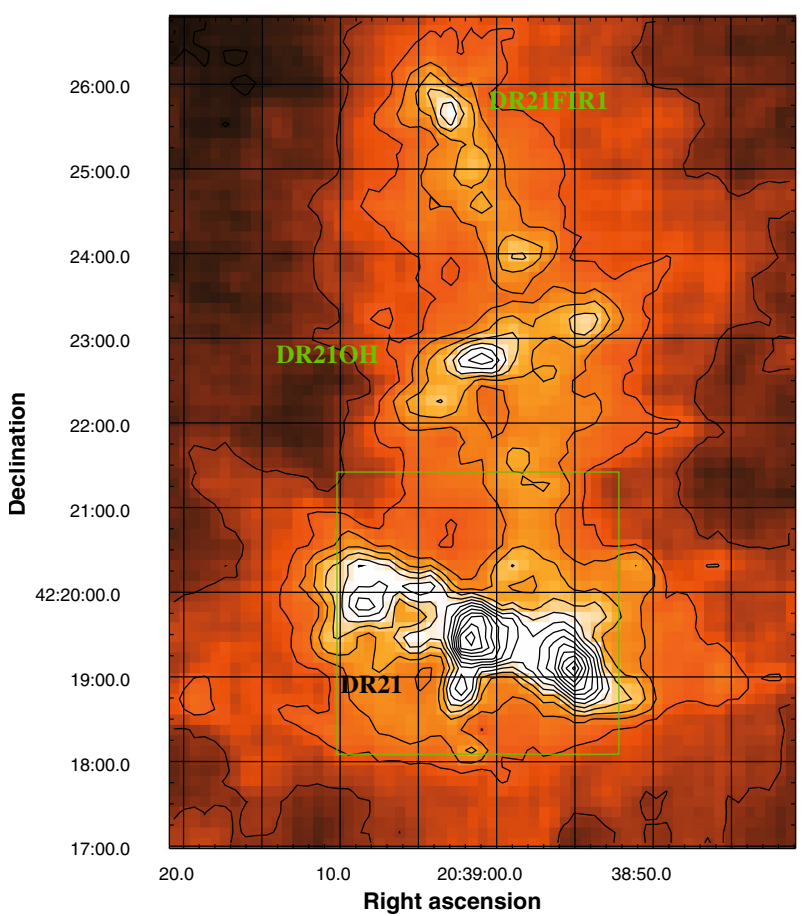

Fig. 3. $\mathrm{CO} J=3-2$ integrated emission map of DR21 between velocities of $-40- \pm 40 \mathrm{~km} \mathrm{~s}^{-1}$. The lowest contour is drawn at $1 \mathrm{~K} \mathrm{~km} \mathrm{~s}^{-1}(5 \sigma$ as observed in a $61 \mathrm{kHz}\left(\sim 0.05 \mathrm{~km} \mathrm{~s}^{-1}\right)$ spectral channel), and the contour intervals are incremented in steps of $50 \mathrm{~K} \mathrm{~km} \mathrm{~s}^{-1}$. The peak antenna temperature and velocity integrated temperatures at the DR21 peak are $39.2 \mathrm{~K}$ and $726 \mathrm{~K} \mathrm{~km} \mathrm{~s}^{-1}$ respectively. The temperature scale is calibrated in antenna temperature $T_{\mathrm{a}}{ }^{*}$ units, which is related to the main beam brightness temperature $T_{\mathrm{mb}}$ by $T_{\mathrm{mb}}=T_{\mathrm{a}}{ }^{*} / \eta_{\mathrm{mb}}$, where the main beam efficiency, $\eta_{\mathrm{mb}}=0.63$. Further details of HARP calibration are given in Buckle et al. (2010).

\section{Modeling the $\mathrm{CO}$ lines}

The most extensive modeling of the CO toward DR21 is by Richardson et al. (1986, 1988), Wilson et al. (1990), Schneider et al. (2006) and Jakob et al. (2007). Richardson et al. (1986, 1988) presented a multiphase model with gas densities spanning the range $10^{3}-10^{6} \mathrm{~cm}^{-3}$, and gas temperatures in the low temperature component $\gtrsim 30 \mathrm{~K}$. Jakob et al. (2007) confirmed this using KOSMA and ISO observations, finding and additional warm phase component with $T_{\text {kin }} \sim 80-150 \mathrm{~K}$ and clump density $n_{\mathrm{H}_{2}} \sim 10^{4}-10^{6} \mathrm{~cm}^{-3}$.

We initially constructed a rotational temperature diagram for the SPIRE CO and ${ }^{13} \mathrm{CO}$ lines. These were augmented with the JCMT CO line from Sect. 2.3, plus IRAM CO $J=2-1$ observations (Schneider et al. 2010), with suitable beam size corrections. The rotational temperature diagram is shown in Fig. 4.

Both species show evidence for two gas components, a lower temperature phase with a rotational temperature $T_{\text {rot }}=78 \mathrm{~K}$ and total CO column density $N(\mathrm{CO}) \sim 4.5 \times 10^{21} \mathrm{~cm}^{-2}$, in addition to a higher temperature component with $T_{\text {rot }}=185 \mathrm{~K}$ and $N(\mathrm{CO}) \sim$ $9.7 \times 10^{17} \mathrm{~cm}^{-2}$. The ${ }^{13} \mathrm{CO}$ lines are more limited and noisy, with the SPIRE lines indicating an intermediate temperature phase having $T_{\text {rot }}=109 \mathrm{~K}$ and $N\left({ }^{13} \mathrm{CO}\right) \sim 8.8 \times 10^{16} \mathrm{~cm}^{-2}$. The data for ${ }^{13} \mathrm{CO}$ also show evidence for a low temperature component, although this relies on comparison with low frequency ground based data (JCMT, IRAM) obtained with different beam sizes. Such a result is expected, since the observations probe deeper into the PDR of each clump in ${ }^{13} \mathrm{CO}$ than in $\mathrm{CO}$.

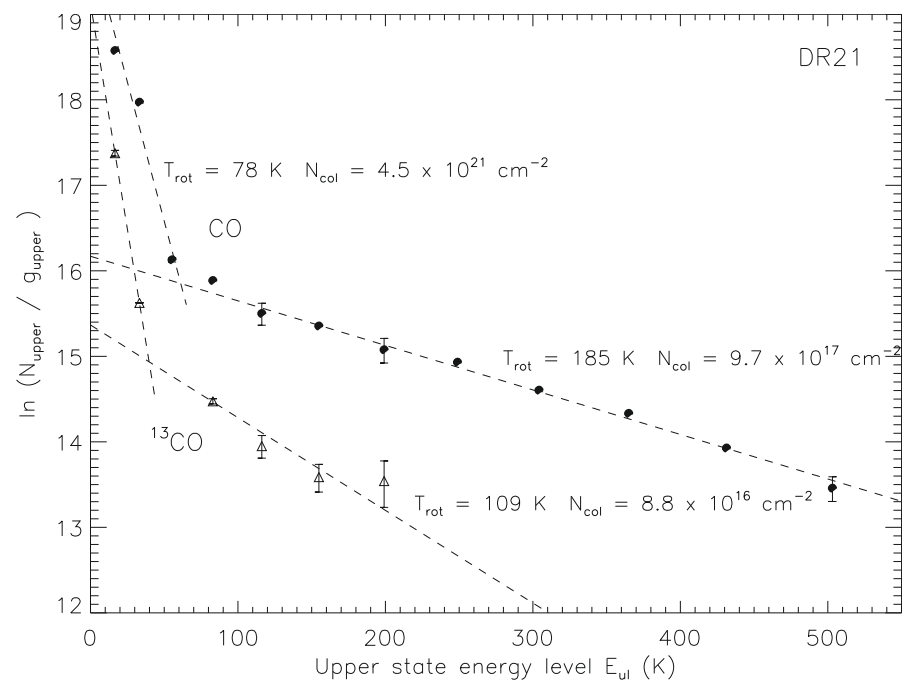

Fig. 4. $\mathrm{CO}$ and ${ }^{13} \mathrm{CO}$ line rotational temperature diagram.

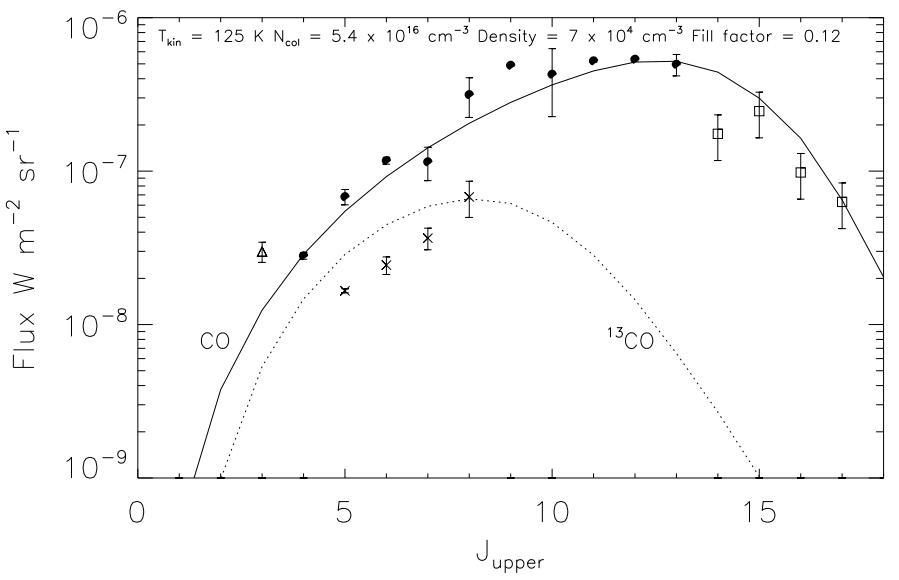

Fig. 5. LVG analysis of the $\mathrm{CO}$ and ${ }^{13} \mathrm{CO}$ lines toward DR21. The SPIRE data points, $(J=4-3$ to $13-12)$ are shown as filled circles, and the ISO data points (Jakob et al. 2007) as squares, along with $30 \%$ absolute error bars. The CO $J=3-2$ line was obtained from the JCMT data, convolved to a resolution of $17^{\prime \prime}$ to match the beamsize of the nearby SPIRE lines. The filled circles are the SPIRE data, open sqares are ISO data from Jakob et al. (2007), open triangle is determined from the JCMT data in this paper, and the crosses are the SPIRE ${ }^{13} \mathrm{CO}$ data. The top solid curve shows the fit to the predicted SPIRE CO line intensities, and the lower dashed one is for ${ }^{13} \mathrm{CO}$ with an assumed $\mathrm{CO} /{ }^{13} \mathrm{CO}$ abundance of 67 , an estimated filling factor of $12 \%$. As might be expected, the fit for a single tempeature lies intermediate between the two $T_{\text {rot }}$ values inferred from Fig. 4.

There are several problems with the rotational temperature approach, including wavelength dependent beam size corrections, opacity and calibration errors. These uncertainties can however be mitigated by i) taking ratios of the various $\mathrm{CO}$ line intensities on a single detector and using these to constrain the excitation conditions though our LVG modeling, and ii) using observations from the central pixel where the SSW and SLW beams are coincident and the calibration is well determined. This approach particularly mitigates against the beam size and calibration errors, since only flux ratios are being used to estimate the excitation conditions.

The model fit was made to the $\mathrm{CO}$ and ${ }^{13} \mathrm{CO}$ lines using the off-line version of the RADEX LVG code (Van der Tak et al. 2007). The line ratios observed on the same detectors (hence the beam sizes are similar) were used to restrict the likely 
excitation conditions. It proved difficult to find an unique single temperature model that simultaneously predicted the relative intensities of both isotopologues. However, the SPIRE data can be approximately reproduced by a single phase moderate temperature gas with $T_{\text {kin }} \sim 125 \mathrm{~K}$, volume density $\sim 7 \times 10^{4} \mathrm{~cm}^{-3}$, with $N(\mathrm{CO}) \sim 3.5 \times 10^{18} \mathrm{~cm}^{-2}$, filling factor $\sim 12 \%$, and a $[\mathrm{C}] /\left[{ }^{13} \mathrm{C}\right]$ ratio of 65 . This model does however slightly overpredict the low- $J{ }^{13} \mathrm{CO} 4-3-6-5$ line intensities, compared to the $J=7-6$ line. Changing the temperature and density from these conditions considerably worsened the high- $J$ CO line fits, although a more complex multiphase model, with appropriate (and uncertain) beam size corrections would improve the fit of the low $J$-lines. We have not attempted to fit to a PDR-model, as the data and calibration quality need to be improved if tests between models are to be made, and that this is beyond this first look paper.

\section{Modeling the $\mathrm{H}_{2} \mathrm{O}$ and [N II] lines}

An objective of this study was to detect the [N II] $205 \mu \mathrm{m}$ line, and to compare it with the $[\mathrm{C} \mathrm{III}]_{157}$ line which has $n_{\text {crit }}=$ $46 \mathrm{~cm}^{-3}, T_{\mathrm{e}}=8000 \mathrm{~K}$. This has a nearly identical critical density for excitation in ionised regions. Their line ratio is directly related to the $\mathrm{N}^{+} / \mathrm{C}^{+}$abundance ratio, and this ratio traces the fraction of the observed [C II] emission that arises from ionized regions (Oberst et al. 2006). Taking the SPIRE upper limit of $7.5 \times 10^{-8} \mathrm{~W} \mathrm{~m}^{-2} \mathrm{sr}^{-1}$ with Jakob et al. (2007), the ratio of the $122 / 205 \mu \mathrm{m}$ lines is $\geq 1.9$, which is only adequate to constrain the ionised gas density to be $\geq \sim 30 \mathrm{~cm}^{-3}$. The $[\mathrm{C} \mathrm{III}] /[\mathrm{N} \mathrm{II}]_{205}$ ratio using the Jakob et al. (2007) tabulation is $\geq 5.6$. Given current uncertainties and lack of an $[\mathrm{N} \mathrm{II}]_{122}$ flux, it is necessary to await improved data. We note that the $[\mathrm{N}$ II] extension to the east (see Fig. 2) coincides with a hole in the excited $\mathrm{H}_{2}$ emission image (Cruz-González et al. 2010), which may indicate there is a cavity of ionised gas. However clarification will require future observations with better sampling.

In Fig. 6 we show a section of the spectrum with the $398.6 \mu \mathrm{m}$ para- $\mathrm{H}_{2} \mathrm{O}$ line, and the $\mathrm{HCO}^{+} J=6-5,7-6$ and 87 lines. Putting the SPIRE sensitivity into perspective, Jakob et al. (2007) report that the integrated $\mathrm{CI}{ }^{3} \mathrm{P}_{1}-{ }^{3} \mathrm{P}_{0}$ intensity measured from the KOSMA telescope with an $80^{\prime \prime}$ beam is $46.6 \mathrm{~K} \mathrm{~km} \mathrm{~s}^{-1}$, and main beam brightness temperature $\sim 25 \mathrm{~K}$. By comparison the same line observed with SPIRE has a peak $S / N$ ratio of $\gtrsim 14$ as seen in a single SPIRE channel. We also used RADEX to compute an LVG solution for the $2_{11}-2_{02}$ para$\mathrm{H}_{2} \mathrm{O}$ line at $398.5 \mu \mathrm{m}$. Assuming similar excitation to that from the $\mathrm{CO}$ solution, for an abundance $X\left[\mathrm{H}_{2} \mathrm{O}\right]=\sim 4 \times 10^{-8}$ and line width of $40 \mathrm{~km} \mathrm{~s}^{-1}$ (Hjalmarson et al. 2003), we predict that the SPIRE flux should be $2.4 \times 10^{-8} \mathrm{~W} \mathrm{~m}^{-2} \mathrm{sr}^{-1}$, which agrees with the measured value of $2.33 \pm 0.3 \times 10^{-8} \mathrm{~W} \mathrm{~m}^{-2} \mathrm{sr}^{-1}$.

\section{Conclusions}

We have presented the SPIRE spectrum of a star-forming molecular core, DR21, showing for the first time the complete CO and ${ }^{13} \mathrm{CO}$ band head from $J=4-3$ to $13-12$, along with their maps at far-infrared wavelengths. A rotational temperature analysis shows two gas phases with $T_{\text {rot }} \sim 80 \mathrm{~K}$ and CO column density $\sim 4.5 \times 10^{18} \mathrm{~cm}^{-2}$, and $T_{\text {rot }}=185 \mathrm{~K}$ and $N(\mathrm{CO}) \sim 10^{18} \mathrm{~cm}^{-2}$

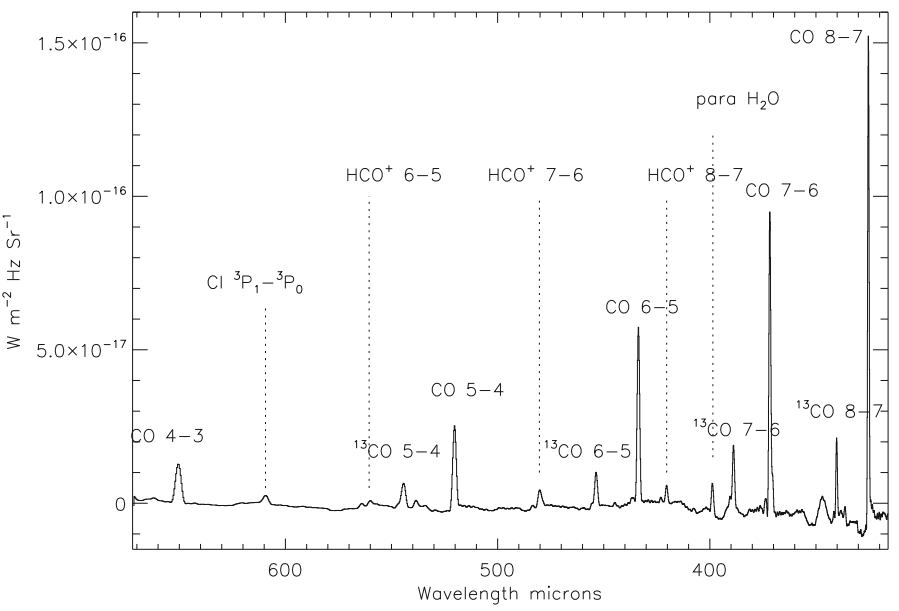

Fig. 6. Continuum subtracted spectrum showing the $398.6 \mu \mathrm{m}$ para$\mathrm{H}_{2} \mathrm{O}$, and $\mathrm{HCO}^{+} J=7-6$ and $J=8-7$ lines. Some instrumental features remain - notably a broad bump close to the $\mathrm{CH}^{+}$line at $347.9 \mu \mathrm{m}$. Although it is possible to flatten this spectrum further, we chose only to fit a third order polynomial to the entire $671-319 \mu \mathrm{m}$ range of the SLW detector.

respectively, although the ${ }^{13} \mathrm{CO} T_{\text {rot }}$ is intermediate between these two. Simple LVG modeling shows the presence of warm $(125 \mathrm{~K})$ and dense $\left(n_{\mathrm{H}_{2}} \sim 7 \times 10^{4} \mathrm{~cm}^{-3}\right)$ gas, which is traced by in the SW extension in the $\mathrm{CO}$ and CI maps. The observed flux from the $398.6 \mu \mathrm{m} \mathrm{H}_{2} \mathrm{O}$ line is consistent with these values.

Acknowledgements. SPIRE has been developed by a consortium of institutes led by Cardiff Univ. (UK) and including Univ. Lethbridge (Canada); NAOC (China); CEA, LAM (France); IFSI, Univ. Padua (Italy); IAC (Spain); Stockholm Observatory (Sweden); Imperial College London, RAL, UCL-MSSL, UKATC, Univ. Sussex (UK); Caltech, JPL, NHSC, Univ. Colorado (USA). This has been supported by national funding agencies: CSA (Canada); NAOC (China); CEA, CNES, CNRS (France); ASI (Italy); MCINN (Spain); Stockholm Observatory (Sweden); STFC (UK); and NASA (USA).

\section{References}

Buckle, J. V., Curtis, E. I., Roberts, J. F., et al. 2010, MNRAS, 401, 204 Cruz-González, I., Salas, L., \& Hiriart, D. 2007, Rev. Mex. Astron. Astrofis, 43, 337

Fixen, D. J., Bennett C. L., \& Mather, J. C. 1999, ApJ, 526, 207

Garden, R., Hayashi, M., Gatley, I., et al. 1991, ApJ, 374, 540

Griffin, M. J., et al. 2010, A\&A, 518, L3

Hjalmarson, A., Frisk, U., Olberg, M., et al. 2003, A\&A, 402, L39

Jakob, H., Kramer, C., Simon, R. et al., 2007, A\&A, 461, 999

Jones, S. C., Naylor, D. A., Gom, B. G., et al. 2009, Proc. 30th Canadian Symposium on Remote Sensing, Canadian Remote Sensing Society, in press Liechti, S., \& Walmsley, C. M. 1997, A\&A, 321, 625

Oberst, T. E., Parshley, S. C., Stacey, G. J., et al. 2006, ApJ, 652, 125 Pilbratt, G. L., et al. 2010, A\&A, 518, L1

Richardson, K. J., White, G. J., et al. 1986, MNRAS, 219, 167, 1986 Richardson, K. J., White, G. J., Monteiro, T., et al. 1988, A\&A, 198, 237 Richardson, K. J., Sandell, G., \& Krisciunas, K. 1989, A\&A, 224, 199

Schneider, N., Bontemps, S., Simon, R., et al. 2006, A\&A, 458, 855

Schneider, N., Csengeri, N., Bontemps, S., et al. 2010, A\&A, accepted [arXiv: 1003.4198]

Swinyard, B. M., et al. 2010, A\&A, 518, L4

Van der Tak, F., Black, J., Schoier, F., et al. 2007, A\&A, 468, 627

Wilson, T. L., \& Mauersberger, R. 1990, A\&A, 239, 305 
1 Department of Physics \& Astronomy, The Open University, UK e-mail: g.j.white@open.ac.uk

2 Space Science Department, Rutherford Appleton Laboratory, Chilton, UK

3 Institute for Space Imaging Science, University of Lethbridge, Lethbridge, Alberta, Canada

${ }^{4}$ Institut d'Astrophysique Spatiale, CNRS/Université Paris-Sud 11, 91405 Orsay, France

5 Laboratoire AIM, CEA/IRFU - CNRS/INSU - Université Paris Diderot, CEA-Saclay, 91191 Gif-sur-Yvette Cedex, France

${ }^{6}$ CEA, Laboratoire AIM, Irfu/SAp, Orme des Merisiers, 91191 Gifsur-Yvette, France

7 Department of Physics and Astronomy, Cardiff University, Cardiff, UK

${ }^{8}$ Laboratoire d'Astrophysique de Marseille, UMR6110 CNRS, 38 rue F. Joliot-Curie, 13388 Marseille, France
9 Canadian Institute for Theoretical Astrophysics, Toronto, Ontario, M5S 3H8, Canada

10 University of California, Radio Astronomy Laboratory, Berkeley, 601 Campbell Hall, US Berkeley CA 94720-3411, USA

11 Institut de Radioastronomie Millimétrique (IRAM), 300 rue de la Piscine, 38406 Saint-Martin-d'Hères, France

12 Joint Astronomy Centre, University Park, Hilo, USA

13 National Astronomical Observatories (China)

14 Istituto di Fisica dello Spazio Interplanetario, INAF, Via del Fosso del Cavaliere 100, 00133 Roma, Italy

15 NASA-Goddard SFC, USA

16 Laboratoire des Signaux et Systèmes (CNRS Supélec Université Paris-Sud 11), Plateau de Moulon, 91192 Gif-sur-Yvette, France

17 Université de Toulouse; UPS; CESR; 9 avenue du colonel Roche, 31028 Toulouse Cedex 4, France

18 CNRS; UMR5187; 31028 Toulouse, France 